\title{
Association between collagen cross-links and trabecular microarchitecture properties of human vertebral bone
}

\author{
S. Viguet-Carrin a,*, H. Follet ${ }^{\text {a }}$, E. Gineyts ${ }^{\text {a }}$, J.P. Roux ${ }^{\text {a }}$, F. Munoz ${ }^{\text {a }}$, R. Chapurlat ${ }^{\text {a }}$, \\ P.D. Delmas ${ }^{\mathrm{a}}$, M.L. Bouxsein ${ }^{\mathrm{b}}$ \\ a INSERM Research Unit 831 and Claude Bernard University of Lyon, Lyon, France \\ b Orthopedic Biomechanics Laboratory, Beth Israel Deaconess Medical Center and Harvard Medical School, Boston, MA, USA
}

\section{A R T I C L E I N F O}

\section{Article history:}

Received 26 June 2009

Revised 29 September 2009

Accepted 1 October 2009

Available online $\mathrm{xxxx}$

Edited by: R. Rizzoli

\section{Keywords:}

Microarchitecture

Collagen cross-links

Pentosidine

Vertebrae

Trabecular bone

\begin{abstract}
A B S T R A C T
It has been suggested that age-related deterioration in trabecular microarchitecture and changes in collagen cross-link concentrations may contribute to skeletal fragility. To further explore this hypothesis, we determined the relationships among trabecular bone volume fraction (BV/TV), microarchitecture, collagen cross-link content, and bone turnover in human vertebral trabecular bone. Trabecular bone specimens from L2 vertebrae were collected from 51 recently deceased donors (54-95 years of age; 20 men and 30 women). Trabecular bone volume and microarchitecture was assessed by microCT and bone formation, reflected by osteoid surface (OS/BS, \%), was measured by 2D histomorphometry. Pyridinoline (PYD), deoxypyridinoline (DPD), pentosidine (PEN) and collagen content in the cancellous bone were analysed by high-performance liquid chromatography. Associations between variables were investigated by Pearson correlations and multiple regression models, which were constructed with BV/TV and collagen cross-links as explanatory variables and microarchitecture parameters as the dependent variables. Results: Microarchitecture parameters were modestly to strongly correlated with BV/TV $\left(r^{2}=0.10-0.71\right)$. The amount of mature enzymatic PYD and DPD cross-links were not associated with the microarchitecture, either before or after adjustment for BV/TV. However, there was a positive correlation between PEN content and trabecular number $(r=0.45, p=0.001)$ and connectivity density $(r=0.40, p=0.004)$, and a negative correlation between PEN content and trabecular separation $(r=-0.29, p=0.04)$. In the multiple regression models including BV/TV, age and PEN content was still significantly associated with several of the microarchitecture variables. In summary, this study suggests a link between trabecular microarchitecture and the collagen cross-link profile. As PEN reflects non-enzymatic glycation of collagen and generally increases with bone age, the association between PEN and trabecular architecture suggests that the preserved trabeculae may contain mainly old bone and have undergone little remodeling. Thus, vertebral fragility may not only be due to alterations in bone architecture but also to modification of collagen cross-link patterns thereby influencing bone's mechanical behavior.
\end{abstract}

(c) 2009 Elsevier Inc. All rights reserved.

\section{Introduction}

In addition to bone mass, other factors may influence trabecular bone strength, including microarchitecture, degree of mineralization, presence of microdamage, and the type and the amount of collagen cross-links [1]. In humans, vertebral bodies are constituted mainly by trabecular bone with relatively low bone volume fraction (BV/TV ranges from $5 \%$ to $30 \%$ ) [2-5]. With such a small amount of trabecular material, an optimal structural organization of the trabeculae is important. Patients with vertebral fractures have significantly deteriorated cancellous bone structure compared with controls matched for bone density [6].

\footnotetext{
* Corresponding author. Nestle Research Center, P.O. Box 44, 1000 Lausanne 26, Switzerland. Fax: +41217858544.

E-mail address: stephanie.viguet-carrin@rdls.nestle.com (S. Viguet-Carrin).
}

Type I collagen, the principal protein of the organic matrix, is organized into fibers. These fibers are stabilized by trivalent mature enzymatic cross-links called pyridinoline (PYD), deoxypyridinoline (DPD) and pyrrole which result in a link between two telopeptides and the helix part of another collagen molecule [7-9]. In addition, a non-enzymatic process leads to the formation of advanced glycation end products (AGEs), some of them forming cross-links between collagen fibers such as pentosidine (PEN) $[10,11]$. Since the initial characterization of the pentosidine cross-link many AGEs (and more recently advanced lipoxidation end products) have been identified $[11,12]$. AGEs occur in tissues characterized by low turnover and consequently containing long-lived proteins, such as collagen in the extracellular matrix of connective tissues (e.g., cartilage, bone, tendon, and skin) $[13,14]$. In contrast to the enzymatic cross-links, reactions resulting in AGE cross-links occur at a late stage when the matrix has been laid down. Once formed, AGEs are removed from the 
tissue only when the protein involved is degraded [15]. It has previously been reported that cross-links influence bone mechanical properties, and especially that accumulation of AGEs may contribute to skeletal fragility with aging [16-19]. However, only one study has analysed the relationship among trivalent mature enzymatic crosslinks, trabecular architecture and bone remodeling [20]. Specifically, Banse et al. [20] reported that the concentration of pyrrole and PYD in human lumbar vertebrae is associated with the trabecular microarchitecture, such that vertebrae having low pyrrole and high PYD contents had relatively more thin trabeculae spread over a complex network. In contrast, specimens with high pyrrole or low PYD content had a relatively thick trabeculae and a simple structure. However, this study investigated only immature and trivalent mature enzymatic cross-links and was limited to 2D microarchitecture assessment by histology. Thus, further investigation of relationship between both enzymatic and non-enzymatic collagen cross-links and trabecular microrchitecture is warranted.

To address these shortcomings, the aims of the present study were to use human vertebral trabecular bone specimens to (1) describe the age-related change in microarchitecture and collagen cross-link characteristics and (2) assess the relationship between cross-links derived from both enzymatic and non-enzymatic processes and the trabecular bone microarchitecture. In particular we asked whether trabecular bone volume and collagen cross-links are associated independently with vertebral trabecular microarchitecture.

\section{Material and methods}

\section{Sample collection}

Lumbar vertebrae (L2) were collected from 51 human cadavers including 20 men $(75.4 \pm 10$ years, mean $\pm S D)$ and 30 women $(80.4 \pm 10$ years, mean \pm SD). The sex of one sample was not known. The age of the donors ranged from 54 to 95 years old. The absence of prevalent fractures or significant bone diseases (e.g., metastasis, Paget's disease, osteochondritis, spondylosis) was confirmed by X-ray radiography of the vertebrae. No additional information regarding donor disease status or medication history was available. A core of trabecular bone, oriented according to the supero-inferior direction of the vertebra was removed from each vertebral body using a diamond tipped coring tool (diameter 8.25 $\mathrm{mm}$; Starlite Industries). Trabecular bone adjacent to the cores was kept frozen at $-20{ }^{\circ} \mathrm{C}$ until collagen cross-link analysis. The cores were kept in $70 \%$ ethanol until bone imaging and histological processing.

\section{Bone imaging}

Bone mineral content (BMC, g) of the vertebral trabecular cores were measured by dual-energy X-ray absorptiometry (pDXA; PIXImus, GE-Lunar Corp., Madison, WI). Volumetric density (vBMD) was then calculated by dividing the BMC by the specimen volume, as measured by a digital caliper. Cores were then imaged by high resolution microtomography ( $\mu \mathrm{CT} 40$; Scanco Medical AG, Basserdorf, Switzerland), with an isotropic resolution of $20 \mu \mathrm{m}$. Bone volume fraction and microarchitecture parameters were computed from the reconstructed, thresholded images using 3D algorithms, as previously described [21]. We quantified the bone volume fraction (BV/TV; \%), trabecular number (Tb.N, $\mathrm{mm}^{-1}$ ), trabecular thickness (Tb.Th, $\mu \mathrm{m})$, trabecular separation (Tb.Sp, $\mu \mathrm{m})$, connectivity density (ConnD., $\mathrm{mm}^{-3}$ ), degree of anisotropy (DA, \#) and the structure model index (SMI, \#), which reflects the rodversus-plate-like nature of the structure. In order to minimize the artifacts due to the coring, the defined volume of interest for morphometric measurements excluded $1 \mathrm{~mm}$ of bone at the periphery of the biopsy.

\section{Bone histomorphometry}

After bone imaging analyses, specimens were dehydrated in 100\% ethanol, substituted in methylcyclohexane and embedded in methymethacrylate using a standard protocol [22]. For the histomorphometric analysis, three 8 - $\mu \mathrm{m}$ sections were cut $200 \mu \mathrm{m}$ apart parallel to the long axis. Bone sections were stained with modified Goldner Trichrome and a semiautomatic analyser (Tablet'measure, ExploraNova, La Rochelle, France) used to assess the ratio of osteoid surface to bone surface (OS/BS, \%).

\section{Biochemical analysis}

To avoid contaminating trabecular bone by other tissues, only the central part of the vertebral body around the coring containing exclusively trabecular bone was retained for biochemical analysis. Trabecular bone samples were cut in small pieces and then defatted with methanol chloroform (1:3). After extensive washing with desionized water, pieces of bone were powdered in a liquid-cooled freeze-mill and lyophilised. Trabecular bone was hydrolysed in $6 \mathrm{~N}$ $\mathrm{HCl}$ for $20 \mathrm{~h}$ at $110^{\circ} \mathrm{C}$. The amount of collagen was determined using hydroxyproline HPLC assay (Bio-rad, Munich, Germany) from an aliquot of bone hydrolysate. Collagen content was calculated on the dry weight of the bone assuming $14 \%$ hydroxyproline in type I collagen. The resulting data were then used to calculate the cross-link values as mmoles per mole of collagen. The content of both mature enzymatic cross-link PYD and DPD, and non-enzymatic cross-links PEN were analysed by reversed-phase HPLC as previously described in detail [23]. Briefly, from an aliquot of the acid hydrolysate in which internal standard (internal standard from Biorad) was added, crosslinks were extracted using solid phase extraction column (Chromabond cross-links ${ }^{\circledR}$, Macherey Nagel, Düren, Germany). Then, PYD, DPD and PEN were separated by HPLC by using an acetonitrile gradient solution containing $0.12 \% n$-heptafluorobutyric acid. PYD and DPD were monitored for fluorescence with excitation at $295 \mathrm{~nm}$ and emission at $395 \mathrm{~nm}$ and then the wavelengths of excitation and emission were shifted to $335 \mathrm{~nm}$ and $385 \mathrm{~nm}$ for detection of PEN. The pyridinium cross-links were quantified against a calibrator supplied by Metra Biosystem Ltd. PEN standard was synthesized [23] and the concentration was calibrated with a standard of pentosidine kindly provided by Dr. Takahashi (University of Saitama, Japan).

\section{Statistical analysis}

Kolmogorov-Smirnov tests were used to test the normality of the distributions, and natural logarithmic transformation was performed for several parameters (collagen content, PEN, Tb.Th, Tb.Sp, OS/BS). Only the collagen content remained non-Gaussian, consequently nonparametric tests were performed for this parameter. After normalization, a Tukey's test (mean $\pm 3 \mathrm{SD}$ ) was applied to exclude the outliers, resulting in exclusion of three values of osteoid surface. Also, values of vBMD from two specimens were excluded from the analysis because a piece of cortical bone, which would artificially inflate the BMC, was observed on the periphery of the core after $\mu \mathrm{CT}$ reconstruction. The other variables from these specimens were within normal ranges and thus only those variables, and not the entire specimen, were excluded. Differences between males and females were investigated by unpaired $t$-tests. Associations between density variables or collagen parameters and trabecular microarchitecture parameters were investigated by Pearson correlations. Finally, relationships among microarchitecture, bone volume fraction, age and collagen characteristics were analysed by multiple regression models. These models were constructed with BV/TV, age and collagen crosslinks as explanatory variables and microarchitecture parameters as the dependent variable. Data are presented as mean \pm standard deviation 
Table 1

Values of age, osteoid surface, collagen characteristics, microarchitecture and density variables measured from male and female human vertebral trabecular bone.

\begin{tabular}{|c|c|c|c|c|c|c|c|c|c|}
\hline & \multicolumn{4}{|c|}{ Overall $(n=51)$} & & & & & \multirow{3}{*}{$p$} \\
\hline & \multirow[b]{2}{*}{ Mean } & \multirow[b]{2}{*}{ Standard deviation } & \multicolumn{2}{|l|}{ Range } & \multicolumn{2}{|c|}{ Women $(n=30)$} & \multicolumn{2}{|c|}{ Men $(n=20)$} & \\
\hline & & & Minimum & Maximum & Mean & Standard deviation & Mean & Standard deviation & \\
\hline Age (years) & 78.4 & 10.1 & 54 & 95 & 80.4 & 10.2 & 75.4 & 9.7 & 0.09 \\
\hline Collagen (\%) & 22.8 & 2.5 & 15.3 & 28.7 & 23.3 & 2.0 & 21.9 & 3.0 & $0.03^{*}$ \\
\hline PYD (mmol/mol coll) & 234.3 & 61.6 & 126 & 406 & 226.2 & 58.1 & 248.5 & 66.6 & NS \\
\hline DPD (mmol/mol coll $)$ & 104.5 & 26.1 & 61 & 192 & 101.5 & 23.2 & 108.5 & 30.4 & NS \\
\hline PEN (mmol/mol coll) & 20.7 & 9.5 & 6.2 & 54.5 & 19.6 & 8.2 & 22.0 & 11.3 & NS \\
\hline OS/BS (\%) & 4.6 & 4.3 & 0.2 & 15.5 & 4.2 & 3.8 & 5.1 & 5.0 & NS \\
\hline BV/TV (\%) & 7.9 & 2.8 & 4.4 & 17.6 & 7.6 & 3.0 & 8.3 & 2.4 & NS \\
\hline Tb.Th $(\mu \mathrm{m})$ & 116.8 & 23.6 & 75.1 & 186.6 & 115.6 & 25.9 & 119.5 & 20.4 & NS \\
\hline Tb.N $\left(\mathrm{mm}^{-1}\right)$ & 0.84 & 0.12 & 0.5 & 1.1 & 0.83 & 0.12 & 0.85 & 0.12 & NS \\
\hline Tb.Sp $(\mu \mathrm{m})$ & 1265.7 & 336.7 & 834 & 2247 & 1337.4 & 366.5 & 1167.9 & 270.8 & 0.08 \\
\hline Conn.D $\left(\mathrm{mm}^{-3}\right)$ & 2.03 & 0.80 & 0.74 & 4.32 & 2.04 & 0.93 & 1.98 & 0.57 & NS \\
\hline DA & 1.68 & 0.20 & 1.28 & 2.36 & 1.67 & 0.23 & 1.68 & 0.17 & NS \\
\hline SMI & 1.77 & 0.49 & 0.33 & 2.52 & 1.83 & 0.51 & 1.69 & 0.48 & NS \\
\hline $\operatorname{vBMD}\left(\mathrm{g} / \mathrm{cm}^{3}\right)$ & 0.08 & 0.03 & 0.03 & 0.16 & 0.07 & 0.03 & 0.09 & 0.03 & $0.02^{*}$ \\
\hline
\end{tabular}

Note. Mann-Whitney test was performed for collagen content (non-Gaussian variable). NS: nonsignificant.

* $p<0.05$ for men vs. women.

(SD) unless otherwise noted. Differences and associations were considered significant if $p$ value $<$ of 0.05 . All statistical analyses were performed using the Statistical Analysis Software (SAS V8; SAS Institute, Cary, NC, USA).

\section{Results}

Influence of sex

Overall, there were no significant differences between men and women, except that women had higher collagen content and lower vBMD than men (Table 1$)$. Also, women tended to be older $(p=0.09)$ and have increased Tb.Sp $(p=0.08)$ compared to men.

\section{Influence of age}

Table 2 shows the mean values of microarchitecture, density and biochemical parameters in the three age groups. As expected, individuals $<75$ years (tertile 1 ) had the highest values of BV/TV and vBMD, as well as the most robust trabecular architecture. Whereas vBMD, BV/TV, TbN, and degree of anisotropy declined with age ( $r=-0.29$ to $-0.53, p<0.05$ for all, Table 3 ), and TbSp and SMI increased with age ( $r=0.53$ and 0.50 , respectively, $p<0.001$, Table 3 ), the tertile analysis showed that most of age-related differences were between the youngest tertile (i.e., tertile 1 ) and the middle and older tertile. There were no differences between the middle and older tertile, suggesting that perhaps the age-related impairment had plateaued. Neither enzymatic cross-link content (PYD, DPD), or nonenzymatic glycation cross-link content (PEN) were associated with age (Table 3). However, PYD and DPD values tended to be lower in the youngest subjects (tertile 1 ).

\section{Relationships among density and microarchitecture parameters}

vBMD and trabecular microarchitecture parameters were moderately to strongly correlated with BV/TV, $r^{2}=0.10-0.76$. (Table 3 ).

Relationships among density, osteoid surface, collagen cross-links and microarchitecture parameters

None of the collagen characteristics correlated with BV/TV, vBMD or OS/BS (Table 3). Enzymatic trivalent mature cross-link content (PYD, DPD) or the combinations of the two cross-links (PYD + DPD, PYD/DPD) were not associated with microarchitecture parameters, neither before or after adjusting for BV/TV. In contrast, there was a positive association between PEN content and Tb.N $(r=0.45$, $p=0.001)$ and connectivity density $(r=0.40, p=0.004)$ and a negative association with Tb.Sp $(r=-0.29, p=0.04)$ (Fig. 1). By using a multiple regression model (Table 4) after adjusting for the contribution of BV/TV and age, PEN content was still significantly

Table 2

Collagen characteristics, osteoid surface, microarchitecture and density parameters as a function of age.

\begin{tabular}{|c|c|c|c|c|}
\hline & Tertile 1 ( $54-75$ years) $(n=17)$ & Tertile 2 ( $76-84$ years) $(n=18)$ & Tertile 3 ( $85-95$ years) $(n=14)$ & ANOVA ( $p$ value) \\
\hline Coll. (\%) & $23.6 \pm 2.1$ & $22.5 \pm 3.1$ & $22.5 \pm 2.0$ & 0.57 \\
\hline PYD ( $\mathrm{mmol} / \mathrm{mol}$ coll $)$ & $218 \pm 77$ & $237 \pm 49$ & $244 \pm 56$ & 0.20 \\
\hline $\mathrm{DPD}(\mathrm{mmol} / \mathrm{mol}$ coll $)$ & $96 \pm 24$ & $113 \pm 31$ & $103 \pm 20$ & 0.17 \\
\hline PEN (mmol/mol coll) & $20.4 \pm 11.5$ & $20.0 \pm 8.4$ & $22.0 \pm 9.4$ & 0.65 \\
\hline OS/BS (\%) & $4.6 \pm 4.0$ & $3.8 \pm 4.1$ & $6.4 \pm 5.1$ & 0.29 \\
\hline BV/TV (\%) & $9.46 \pm 2.4^{\mathrm{a}, \mathrm{b}}$ & $7.06 \pm 2.4$ & $7.08 \pm 3.3$ & 0.003 \\
\hline Tb.Th $(\mu \mathrm{m})$ & $121 \pm 20$ & $117 \pm 30$ & $111 \pm 20$ & 0.31 \\
\hline Tb.N $\left(\mathrm{mm}^{-1}\right)$ & $0.88 \pm 0.11$ & $0.82 \pm 0.13$ & $0.81 \pm 0.12$ & 0.25 \\
\hline Tb.Sp $(\mu \mathrm{m})$ & $1096 \pm 180^{\mathrm{a}, \mathrm{b}}$ & $1290 \pm 349$ & $1461 \pm 388$ & 0.008 \\
\hline Conn.D $\left(\mathrm{mm}^{-3}\right)$ & $2.11 \pm 0.71$ & $1.96 \pm 0.79$ & $2.01 \pm 1.01$ & 0.73 \\
\hline $\mathrm{DA}(\#)$ & $1.75 \pm 0.17^{\mathrm{b}}$ & $1.79 \pm 0.24^{\mathrm{b}}$ & $1.56 \pm 0.16$ & 0.03 \\
\hline SMI (\#) & $1.45 \pm 0.46^{\mathrm{a}, \mathrm{b}}$ & $1.88 \pm 0.40$ & $2.01 \pm 0.49$ & 0.002 \\
\hline $\operatorname{vBMD}\left(\mathrm{g} / \mathrm{cm}^{3}\right)$ & $0.101 \pm 0.020^{\mathrm{a}, \mathrm{b}}$ & $0.076 \pm 0.031$ & $0.069 \pm 0.027$ & 0.005 \\
\hline
\end{tabular}

Note. Mann-Whitney test was performed for collagen content (non-Gaussian distribution).

a Statistically significant difference compared with tertile $2(p<0.05)$.

b Statistically significant difference compared with tertile $3(p<0.05)$. 
Table 3

Correlation coefficients between age, collagen characteristics, osteoid surface, microarchitecture and density variables measured from human vertebral trabecular bone.

\begin{tabular}{|c|c|c|c|c|c|c|c|c|c|c|c|c|c|c|}
\hline & & Age & PYD & DPD & PEN & Collagen & $\mathrm{BV} / \mathrm{TV}$ & Tb.Th & Tb.N & Tb.Sp & Conn.D & DA & SMI & vBMD \\
\hline Age & $(n=50)$ & - & & & & & & & & & & & & \\
\hline Collagen & $(n=51)$ & 0.004 & & & & & & & & & & & & \\
\hline PYD & $(n=51)$ & 0.25 & - & & & & & & & & & & & \\
\hline DPD & $(n=51)$ & 0.07 & $0.52^{* *}$ & - & & & & & & & & & & \\
\hline PEN & $(n=51)$ & 0.15 & -0.2 & -0.04 & - & & & & & & & & & \\
\hline OS/BS & $(n=48)$ & 0.05 & -0.02 & -0.25 & -0.18 & 0.08 & & & & & & & & \\
\hline $\mathrm{BV} / \mathrm{TV}$ & $(n=51)$ & $-0.43^{*}$ & -0.08 & -0.06 & 0.10 & 0.06 & - & & & & & & & \\
\hline Tb.Th & $(n=51)$ & -0.16 & 0.10 & 0.18 & 0.09 & -0.17 & $0.34^{*}$ & - & & & & & & \\
\hline Tb.N & $(n=51)$ & $-0.29^{*}$ & -0.06 & -0.02 & $0.45^{* *}$ & -0.12 & $0.71^{* *}$ & -0.06 & - & & & & & \\
\hline Tb.Sp & $(n=51)$ & $0.53^{* *}$ & 0.07 & -0.05 & $-0.29^{*}$ & 0.006 & $-0.76^{* *}$ & -0.22 & $-0.82^{* *}$ & - & & & & \\
\hline Conn.D & $(n=51)$ & -0.14 & -0.03 & -0.01 & $0.40^{* *}$ & 0.06 & $0.59^{* *}$ & -0.05 & $0.83^{* *}$ & $-0.72^{* *}$ & - & & & \\
\hline $\mathrm{DA}$ & $(n=51)$ & $-0.31^{*}$ & -0.25 & -0.17 & -0.19 & 0.21 & $0.32^{*}$ & 0.06 & -0.08 & -0.09 & -0.26 & - & & \\
\hline SMI & $(n=51)$ & $0.50^{* *}$ & 0.02 & -0.13 & 0.13 & -0.09 & $-0.84^{* *}$ & -0.16 & $-0.44^{* *}$ & $0.60^{* *}$ & $-0.34^{*}$ & $-0.53^{* *}$ & - & \\
\hline vBMD & $(n=49)$ & $-0.53^{* *}$ & -0.17 & -0.14 & 0.13 & -0.03 & $0.87^{* *}$ & 0.23 & $0.61^{* *}$ & $-0.75^{* *}$ & $0.42^{* *}$ & $0.33^{*}$ & $-0.69^{* *}$ & - \\
\hline
\end{tabular}

Note. Pearson correlation coefficients for all parameters except for collagen (Spearman correlations).

$* \quad p \leq 0.05$.

** $p<0.001$.

associated with Tb.N $\left(R^{2}=0.65, p<0.0001\right)$, Tb.Sp $\left(R^{2}=0.70, p<\right.$ $0.0001)$, connectivity density $\left(R^{2}=0.47, p<0.0001\right)$ and SMI $\left(R^{2}=\right.$ $0.79, p<0.0001)$.

\section{Discussion}

In this study we asked whether collagen cross-link content, including enzymatic and non-enzymatic cross-links, was associated with vertebral trabecular microarchitecture. We found that independent of trabecular bone volume, PEN content was positively associated with trabecular number, connectivity density, and structure model index; and negatively associated with trabecular separation. Thus, PEN is associated with denser and rod-like, rather than plate-like trabecular network. This finding implies that the preserved trabeculae contains mostly aged bone that has undergone little remodeling and thus contains higher amounts of AGE cross-links such as PEN. In contrast, the contents of enzymatic PYD and DPD cross-links were not related to the microarchitecture parameters nor were combinations of the two (PYD + DPD, PYD/DPD), either before or after adjustment for $\mathrm{BV} / \mathrm{TV}$.

This study highlights an association between PEN content and bone microarchitecture. The PEN concentrations reported here are comparable to those previously published for human femurs and vertebrae $[17,19,24]$. In our study, PEN content (a marker of nonenzymatic glycation induced cross-links) did not increase with aging. This is in contrast to previous studies that have reported an agerelated increase in AGEs [18,24,25]. However, these previous studies were performed in subjects with a wide age range (0-80 years old) which likely enhanced the ability to detect age-related differences. To illustrate this, we analysed a vertebra from a 26-year-old donor (not included in this study) and found that it contained 7-fold lower PEN content when compared to the mean PEN content of the elderly donors in our current study. This is in agreement with Hernandez et al. [19] who also found no increase in PEN content with age in human vertebral trabecular bone in the same age range (54-94 years old) as our specimens. Altogether, these findings suggest that the age-related increase in PEN content occurs mainly before the 6th decade.

Our data indicate that the degree of non-enzymatic glycation is related to the microarchitecture of vertebral trabecular bone. In contrast to the enzymatic cross-links which occur during formation of the collagen fiber matrix, the glycation reaction resulting in AGE cross-links occurs at a late stage when the matrix has been laid down. Indeed, the low bone turnover during aging allows the accumulation of the products of the Maillard reaction of glucose, some of which form intermolecular cross-links. Although AGEs seem hardly likely to be involved in determining the microarchitecture of bone, the evidence that AGEs accumulate in tissues with low turnover such as bone, cartilage and tendon [26], has led to studies on the effects of AGEs on osteoblast and osteoclast behavior and the relationship between tissue turnover and AGE accumulation. At the cellular level, it has been shown that AGEs and more particularly PEN, inhibit osteoblastic proliferation and differentiation [27-29] while increasing or decreasing osteoclastic bone resorption [30]. An increase in osteoclastic bone resorption, combined with decreased bone formation, indicates that AGEs will not accumulate in vivo. In contrast, in cases of low bone turnover characterized by reductions in both resorption and formation will result in reducing AGEs removal, and consequently increasing their accumulation.

Furthermore, the glycation of collagen may impact bone turnover by modifying the reactivity of the collagen fibers with cell receptors and other matrix components. Indeed, Paul and Bailey have reported that the specific modification by glycation of arginine residues of type I collagen involved in integrin mediated cell attachment reduces both the adhesion and spreading of the osteosarcoma cell line MG-63 [31].
A

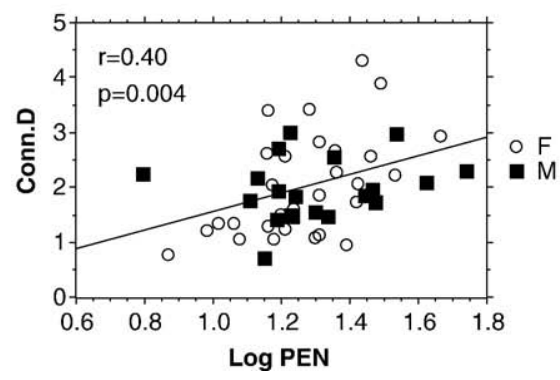

B

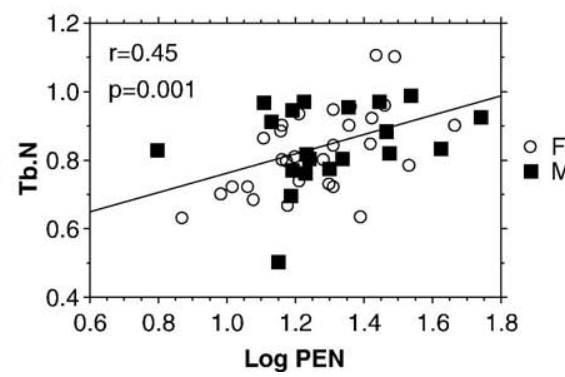

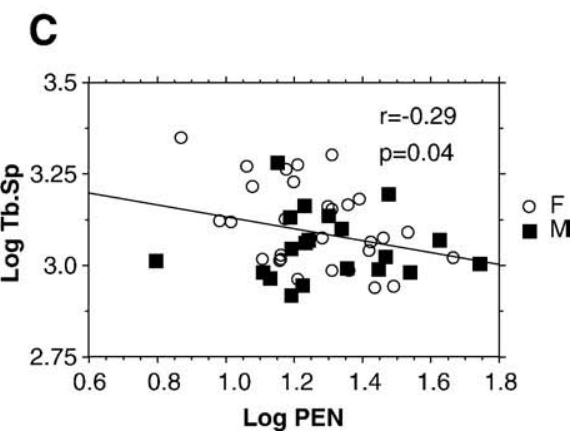

Fig. 1. Correlations between Conn.D (A), Tb.N (B), Tb.Sp (C) and pentosidine measured from human cadaver donors. 
Table 4

Multiple predictions of microarchitecture parameters (Tb.N, Tb.Sp, Conn.D and SMI) from trabecular bone by BV/TV, age and pentosidine.

\begin{tabular}{|c|c|c|c|c|}
\hline \multicolumn{2}{|l|}{ Variable } & \multirow[t]{2}{*}{ Final $R^{2}$} & \multirow{2}{*}{$\begin{array}{l}\text { Standardized } \\
\text { coefficient }(\beta)\end{array}$} & \multirow[t]{2}{*}{$p$} \\
\hline Dependent & Independent & & & \\
\hline \multirow[t]{4}{*}{ Tb.N } & $\mathrm{BV} / \mathrm{TV}$ & & 0.027 & $<0.0001$ \\
\hline & Age & & -0.001 & 0.41 \\
\hline & Log PEN & & 0.25 & $<0.0001$ \\
\hline & & 0.65 & & $<0.0001$ \\
\hline \multirow[t]{4}{*}{ Log Tb.Sp } & $\mathrm{BV} / \mathrm{TV}$ & & -0.023 & $<0.0001$ \\
\hline & Age & & 0.003 & 0.001 \\
\hline & Log PEN & & -0.16 & 0.002 \\
\hline & & 0.70 & & $<0.0001$ \\
\hline \multirow[t]{4}{*}{ Conn.D } & $\mathrm{BV} / \mathrm{TV}$ & & 0.16 & $<0.0001$ \\
\hline & Age & & 0.004 & 0.67 \\
\hline & Log PEN & & 1.42 & 0.004 \\
\hline & & 0.47 & & $<0.0001$ \\
\hline \multirow[t]{4}{*}{ SMI } & $\mathrm{BV} / \mathrm{TV}$ & & -0.15 & $<0.0001$ \\
\hline & Age & & 0.006 & 0.14 \\
\hline & Log PEN & & 0.53 & 0.007 \\
\hline & & 0.79 & & $<0.0001$ \\
\hline
\end{tabular}

The table shows for each model the standardized coefficient of the regression of each variable $(\beta)$, the associated $p$ value, and the square of the final correlation coefficient $\left(R^{2}\right)$.

Thus, the accumulation of AGEs in bone matrix may modify the cell behavior and consequently influence bone remodeling. Moreover, in vivo studies have reported a relationship between the accumulation of AGEs in bone matrix and bone turnover reduction after bisphosphonate therapy. Indeed, the accumulation of AGEs with reduced bone turnover seen in bisphosphonate-treated animals, and in aging humans seems to support this hypothesis. Two studies reported a significant negative correlation between PEN content and the activation frequency in young dogs treated with different bisphosphonates [32,33]. The accumulation of PEN throughout the bone matrix may be due to the prolonged lifetime of bone structural units, caused by the decrease in bone remodeling during bisphosphonate treatments. Moreover, in a recent study, Saito et al. [34,41] used human femur cortical and trabecular bone to demonstrate that PEN accumulates more in older, mineralized osteons compared to younger osteons. The present findings would suggest that an increase in AGEs may not just be a result of tissue aging in situ, but a consequence of alterations in bone remodeling.

In addition to the AGEs, we also investigated the association between trivalent mature cross-links and bone microarchitecture. We found concentrations of PYD and DPD similar to those previously reported in human femurs and vertebrae $[17,24,35]$. Furthermore, the content of PYD tends to increase in elderly subjects, whereas DPD content showed no age-related changes. The slight increase in PYD rather than DPD content may be due to increased lysine hydroxylation. This age-related modification was already reported by Bailey et al. [36] in iliac trabecular human bone.

Only one prior study has investigated the association between collagen characteristics and human vertebral trabecular microrchitecture. Banse et al. [20] found that the pyrrole/PYD ratio reflects the structural organization of the trabeculae. They collected three vertebral bodies (T9; T12-L1 and L4) from nine different subjects (six women and three men; ages 44-88). Several samples were taken from each vertebral body to obtain 68 trabecular bone specimens. Then, they quantified immature and trivalent mature enzymatic cross-links PYD, DPD, pyrrole and collagen content, but not AGEs in the vertebral specimens. In parallel, they analysed the microarchitecture and measured bone formation parameters such as osteoid surface by histomorphometry. They found that bone biopsies with high pyrrole and low PYD content had relatively thick and disconnected trabecular architecture. Inversely, those with low pyrrole and high PYD content had relatively thin trabeculae which were more numerous and spread over a complex network. In our study using only mature pyrididinium cross-links, we observed no association between PYD and vertebral trabecular microarchitecture but we found interesting relationships between PEN and bone microarchitecture. The discrepancies between the two studies may be explained by several methodological differences: (1) Banse et al. quantified immature and mature cross-links, whereas we quantified only the mature enzymatic cross-links; (2) we used only L2 vertebral trabecular specimen whereas lumbar and thoracic vertebrae were used in Banse's study; and (3) Banse et al. used histomorphometry to analyse the 2D microarchitecture, whereas in our study, the microarchitecture was evaluated by 3D $\mu \mathrm{CT}$.

Although we expected that bone remodeling would be related to collagen cross-link pattern and trabecular microstructure, there was no association between osteoid surface, an index of bone formation, and collagen cross-link content. However, this absence of correlation was also reported in human vertebral cores and iliac crests from osteoporotic patients [20,37]. This lack of association may be attributed to the fact that due to specimen origin (which were not double labeled before subject death and not fixed to preserve cellularity) we were unable to analyse all formation and resorption parameters including activation frequency, bone formation rate, and the number of osteoblasts and osteoclasts. Furthermore, our crosssectional sample of middle age to elderly donors may have had too narrow of an age range to see significant age changes, which may be more clearly observed between the young and old, rather than between the middle aged and old. Indeed, OS/BS did not reflect bone turnover, especially not in these elderly subjects who may have had subtle mineralization defects from vitamin D insufficiency or other potential underlying diseases.

This study has both strengths and limitations. A strength of our study was that we used human vertebral specimens from a relatively large number of donors $(n=51)$ and performed both the structural organization and biochemical analyses on the same samples, allowing the analysis of the relationship between collagen characteristics of human vertebral trabecular bone and the microarchitecture of the trabeculae. However, this study also had several limitations. First, we did not have access to the clinical situation before death or the cause of the death. Furthermore, as previously mentioned we could not measure the full complement of histomorphometric variables and immature cross-links and thus, we could not examine all possible associations between collagen cross-links, microstructure, bone cellularity and bone remodeling. Lastly, PEN is just one of many AGEs that accumulates with tissue age. We quantified PEN content in bone tissue because it forms covalent cross-links between adjacent collagen molecules $[12,23]$ and can be accurately measured. However, it is not yet known whether the change in PEN content (one pentosidine cross-link per 50/100 collagen molecules) quantitatively reflects the content of other more abundant AGEs such as glucosepane [38-40], or whether an alternative AGE may have a greater impact on bone microarchitecture variables.

In conclusion, the relationship between bone microarchitecture and collagen cross-link characteristics is complex. Our data suggest an association between the microarchitecture of the trabeculae and the nature of the cross-links that compose the bone matrix. There was no association between trivalent mature enzymatic cross-links and microarchitecture with or without BV/TV adjustment. However, we did find significant associations between the non-enzymatic crosslink pentosidine and microarchitecture parameters. PEN content is associated with dense and more complex rod-like trabecular architecture, independently of bone volume fraction. As pentosidine reflects collagen glycation and generally increases with bone age, the association between pentosidine and trabecular architecture suggests that the preserved trabeculae contain primarily old bone and have undergone little remodeling. Although glycation reactions resulting in AGE cross-links occur at a late stage when the matrix has been laid 
down, the accumulation of these kinds of cross-links may qualitatively influence bone remodeling and therefore alter the final architecture of vertebral trabecular bone. Thus, vertebral fragility may not only be due to the deterioration of bone architecture, but we speculate it may also be due to the modification of collagen cross-link patterns thereby influencing bone mechanical behavior.

\section{Acknowledgments}

We thank C. Bertholon for her technical help for collagen cross-link analysis. This study was supported in part by an unrestricted educational grant from Eli Lilly to INSERM.

\section{References}

[1] Seeman E, Delmas PD. Bone quality - the material and structural basis of bone strength and fragility. N Engl J Med 2006;354:2250-61.

[2] Kleerekoper M, Villanueva AR, Stanciu J, Rao DS, Parfitt AM. The role of threedimensional trabecular microstructure in the pathogenesis of vertebral compression fractures. Calcif Tissue Int 1985;37:594-7.

[3] Bouxsein ML, Melton III LJ, Riggs BL, Muller J, Atkinson EJ, Oberg AL, et al. Age- and sex-specific differences in the factor of risk for vertebral fracture: a populationbased study using QCT. J Bone Miner Res 2006;21:1475-82.

[4] Chen H, Shoumura S, Emura S, Bunai Y. Regional variations of vertebral trabecular bone microstructure with age and gender. Osteoporos Int 2008;19:1473-83.

[5] Lochmuller EM, Matsuura M, Bauer J, Hitzl W, Link TM, Muller R, et al. Site-specific deterioration of trabecular bone architecture in men and women with advancing age. J Bone Miner Res 2008;23:1964-73.

[6] Gordon CL, Lang TF, Augat P, Genant HK. Image-based assessment of spinal trabecular bone structure from high-resolution CT images. Osteoporos Int 1998;8: 317-25.

[7] Eyre D. Collagen cross-linking amino acids. Methods Enzymol 1987;144:115-39.

[8] Knott L, Bailey AJ. Collagen cross-links in mineralizing tissues: a review of their chemistry, function, and clinical relevance. Bone 1998;22:181-7.

[9] Viguet-Carrin S, Garnero P, Delmas PD. The role of collagen in bone strength. Osteoporos Int 2006;17:319-36.

[10] Grandhee SK, Monnier VM. Mechanism of formation of the Maillard protein crosslink pentosidine. Glucose, fructose, and ascorbate as pentosidine precursors. J Biol Chem 1991;266:11649-53.

[11] Sell DR, Monnier VM. Isolation, purification and partial characterization of novel fluorophores from aging human insoluble collagen-rich tissue. Connect Tissue Res 1989;19:77-92.

[12] Sell DR, Monnier VM. Structure elucidation of a senescence cross-link from human extracellular matrix. Implication of pentoses in the aging process. J Biol Chem 1989;264:21597-602.

[13] Bailey AJ, Paul RG, Knott L. Mechanisms of maturation and ageing of collagen. Mech Ageing Dev 1998;106:1-56.

[14] Verzijl N, DeGroot J, Thorpe SR, Bank RA, Shaw JN, Lyons TJ, et al. Effect of collagen turnover on the accumulation of advanced glycation end products. J Biol Chem 2000;275:39027-31.

[15] Avery NC, Bailey AJ. Enzymic and non-enzymic cross-linking mechanisms in relation to turnover of collagen: relevance to aging and exercise. Scand J Med Sci Sports 2005;15:231-40.

[16] Vashishth D, Gibson GJ, Khoury JI, Schaffler MB, Kimura J, Fyhrie DP. Influence of nonenzymatic glycation on biomechanical properties of cortical bone. Bone 2001;28:195-201.

[17] Viguet-Carrin S, Roux JP, Arlot ME, Merabet Z, Leeming DJ, Byrjalsen I, et al. Contribution of the advanced glycation end product pentosidine and of maturation of type I collagen to compressive biomechanical properties of human lumbar vertebrae. Bone 2006;39:1073-9.

[18] Wang X, Shen X, Li X, Agrawal CM. Age-related changes in the collagen network and toughness of bone. Bone 2002;31:1-7.

[19] Hernandez CJ, Tang SY, Baumbach BM, Hwu PB, Sakkee AN, van der HF, et al. Trabecular microfracture and the influence of pyridinium and non-enzymatic glycation-mediated collagen cross-links. Bone 2005;37:825-32.
[20] Banse X, Devogelaer JP, Lafosse A, Sims TJ, Grynpas M, Bailey AJ. Cross-link profile of bone collagen correlates with structural organization of trabeculae. Bone 2002;31:70-6.

[21] Arlot ME, Burt-Pichat B, Roux JP, Vashishth D, Bouxsein ML, Delmas PD. Microarchitecture influences microdamage accumulation in human vertebral trabecular bone. J Bone Miner Res 2008;23:1613-8.

[22] Chavassieux PM, Arlot ME, Reda C, Wei L, Yates AJ, Meunier PJ. Histomorphometric assessment of the long-term effects of alendronate on bone quality and remodeling in patients with osteoporosis. J Clin Invest 1997;100:1475-80.

[23] Viguet-Carrin S, Gineyts E, Bertholon C, Delmas PD. Simple and sensitive method for quantification of fluorescent enzymatic mature and senescent crosslinks of collagen in bone hydrolysate using single-column high performance liquid chromatography. J Chromatogr B Analyt Technol Biomed Life Sci 2009;877:1-7.

[24] Saito M, Marumo K, Fujii K, Ishioka N. Single-column high-performance liquid chromatographic-fluorescence detection of immature, mature, and senescent cross-links of collagen. Anal Biochem 1997;253:26-32.

[25] Tang SY, Allen MR, Phipps R, Burr DB, Vashishth D. Changes in non-enzymatic glycation and its association with altered mechanical properties following 1year treatment with risedronate or alendronate. Osteoporos Int 2009;20: 887-94.

[26] DeGroot J, Verzijl N, Wenting-Van Wijk MJ, Bank RA, Lafeber FP, Bijlsma JW, et al. Age-related decrease in susceptibility of human articular cartilage to matrix metalloproteinase-mediated degradation: the role of advanced glycation end products. Arthritis Rheum 2001;44:2562-71.

[27] Sanguineti R, Storace D, Monacelli F, Federici A, Odetti P. Pentosidine effects on human osteoblasts in vitro. Ann NY Acad Sci 2008;1126:166-72.

[28] Mercer N, Ahmed H, Etcheverry SB, Vasta GR, Cortizo AM. Regulation of advanced glycation end product (AGE) receptors and apoptosis by AGEs in osteoblast-like cells. Mol Cell Biochem 2007;306:87-94.

[29] McCarthy AD, Etcheverry SB, Bruzzone L, Cortizo AM. Effects of advanced glycation end-products on the proliferation and differentiation of osteoblast-like cells. Mol Cell Biochem 1997;170:43-51.

[30] Valcourt U, Merle B, Gineyts E, Viguet-Carrin S, Delmas PD, Garnero P. Nonenzymatic glycation of bone collagen modifies osteoclastic activity and differentiation. J Biol Chem 2007;282:5691-703.

[31] Paul RG, Bailey AJ. The effect of advanced glycation end-product formation upon cell-matrix interactions. Int J Biochem Cell Biol 1999;31:653-60.

[32] Allen MR, Gineyts E, Leeming DJ, Burr DB, Delmas PD. Bisphosphonates alte trabecular bone collagen cross-linking and isomerization in beagle dog vertebra. Osteoporos Int 2008;19:329-37.

[33] Saito M, Mori S, Mashiba T, Komatsubara S, Marumo K. Collagen maturity, glycation induced-pentosidine, and mineralization are increased following 3-year treatment with incadronate in dogs. Osteoporos Int 2008;19:1343-54.

[34] Saito M, Fujii K, Soshi S, Tanaka T. Reductions in degree of mineralization and enzymatic collagen cross-links and increases in glycation-induced pentosidine in the femoral neck cortex in cases of femoral neck fracture. Osteoporos Int 2006;17: 986-95.

[35] Eyre DR, Dickson IR, Van NK. Collagen cross-linking in human bone and articular cartilage. Age-related changes in the content of mature hydroxypyridinium residues. Biochem J 1988;252:495-500.

[36] Bailey AJ, Sims TJ, Ebbesen EN, Mansell JP, Thomsen JS, Mosekilde L. Age-related changes in the biochemical properties of human cancellous bone collagen: relationship to bone strength. Calcif Tissue Int 1999;65:203-10.

[37] Hein G, Weiss C, Lehmann G, Niwa T, Stein G, Franke S. Advanced glycation end product modification of bone proteins and bone remodelling: hypothesis and preliminary immunohistochemical findings. Ann Rheum Dis 2006;65:101-4.

[38] Monnier VM, Mustata GT, Biemel KL, Reihl O, Lederer MO, Zhenyu D, et al. Crosslinking of the extracellular matrix by the maillard reaction in aging and diabetes: an update on "a puzzle nearing resolution". Ann N Y Acad Sci 2005; 1043:533-44.

[39] Sell DR, Biemel KM, Reihl O, Lederer MO, Strauch CM, Monnier VM. Glucosepane is a major protein cross-link of the senescent human extracellular matrix. Relationship with diabetes. J Biol Chem 2005;280:12310-5.

[40] Biemel KM, Friedl DA, Lederer MO. Identification and quantification of major maillard cross-links in human serum albumin and lens protein. Evidence for glucosepane as the dominant compound. J Biol Chem 2002;277:24907-15.

[41] Saito M, Fujii K, Marumo K. Degree of mineralization-related collagen crosslinking in the femoral neck cancellous bone in cases of hip fracture and controls. Calcif Tissue Int 2006;79:160-8. 